\title{
Conocimientos y prácticas asociadas al cumplimiento de la ley que regula el régimen jurídico de canes (Ley Nro. 27596) en los distritos de Lima, Perú
}

\author{
Knowledge and practices associated with compliance with the law that regulates \\ the legal regime of dogs $\left(\operatorname{Law} N .^{\circ} 27596\right)$ in the districts of Lima, Peru
}

\author{
José Llaja1, Daphne León', Néstor Falcónn ${ }^{1,2}$
}

\section{Resumen}

El objetivo del estudio fue evaluar los conocimientos y prácticas asociados al cumplimiento de la Ley N. ${ }^{\circ}$ 27596, Ley que Regula el Régimen Jurídico de Canes, en pobladores de distritos de Lima Metropolitana. Se diseñó y validó una encuesta que incluyó variables sobre conocimientos y prácticas asociadas a los principales aspectos normativos de la mencionada ley, y se aplicó en espacios públicos a tenedores de canes de 20 distritos de estratos socioeconómicos alto, medio y bajo. La participación en el estudio fue voluntaria, obteniéndose 783 encuestas. El 67.6\% conocía que existían razas potencialmente peligrosas (nombraron a Pitbull y Rottweiler) y el $24.9 \%$ conocía la existencia de requisitos para su tenencia. El 35.1\% afirmó que la castración disminuía la agresividad de los canes y $24.8 \%$ opinaron que los canes deben de ser adiestrados en centros especializados. El 95\% afirmó que el dueño del can debe asumir los daños que produce su can, el 29.4\% sabía que los dueños de canes de razas potencialmente peligrosas deberían tener un seguro contra accidentes y el 58.7\% reconocía que la Policía Nacional debería auxiliar a las víctimas de mordedura de canes. El 12.8\% reconoció que sus canes habían mordido y el $72.0 \%$ asumió los gastos ocasionados por su can. La práctica de recojo de heces y paseo de canes fue mayoritaria, pero la de identificación, registro en la municipalidad y control reproductivo de canes fue limitada. El 14.3\% afirmó haber recibido charlas de tenencia responsable de animales de compañía, 16.2\% escuchó sobre la Ley N. ${ }^{\circ} 27596$ y el 8.4\% sabía que existía una Ordenanza Municipal con relación a dicha ley en su distrito. Se hace necesario una mayor difusión de la Ley N. ${ }^{\circ} 27596$ y así como una exigencia para su aplicación.

Palabras clave: perro, salud pública, rabia, zoonosis

${ }^{1}$ Laboratorio de Epidemiología y Salud Pública en Veterinaria, Facultad de Medicina Veterinaria y Zootecnia, Universidad Peruana Cayetano Heredia, Lima, Perú

${ }^{2}$ E-mail: nestorfalcon@upch.pe

Recibido: 9 de agosto de 2020

Aceptado para publicación: 23 de enero de 2021

Publicado: 24 de abril de 2021 
The aim of this study was to evaluate the knowledge and practices associated with compliance with Law $\mathrm{N}^{\circ} 27596$, law that regulates the legal regime of dogs, among the population of districts of Lima, Perú. A survey was designed and validated that included variables on knowledge and practices associated with the main regulatory aspects of the law. The survey was applied in public spaces to dog owners from 20 districts of high, medium and low socioeconomic status. Participation in the study was voluntary, obtaining 783 surveys. In general, $67.6 \%$ knew about potentially dangerous breeds (they named Pitbull and Rottweiler) and $24.9 \%$ knew about the existence of requirements for their possession. Besides, $35.1 \%$ said that castration decreased the aggressiveness of the dogs and $24.8 \%$ believed that the dogs should be trained in specialized centers, $95 \%$ affirmed that the owner of the dog must assume the damages that his dog produces, $29.4 \%$ knew that the owners of dogs of potentially dangerous breeds should have insurance against accidents and 58.7\% recognized that the National Police should help the dog bite victims. Some acknowledged that their dogs had bitten (12.8\%) and $72.0 \%$ assumed the expenses caused by their dogs. The practice of collecting faeces and walking the dogs was the majority, but that of identification, registration in the municipality and reproductive control of dogs was limited. Moreover, $14.3 \%$ claimed to have received talks on responsible pet ownership, 16.2\% heard about Law $\mathrm{N}^{\circ} 27596$ and $8.4 \%$ knew that there was a Municipal Ordinance in relation to that law in their district. It is necessary a greater dissemination of Law $\mathrm{N}^{\circ} 27596$ as well as a requirement for its application.

Key words: dog, public health, rabies, zoonoses

\section{INTRODUCCIÓN}

Los animales de compañía juegan un rol importante en la sociedad debido a que contribuyen en el desarrollo físico, social y emocional de las personas, especialmente en los niños (Robertson et al., 2000). Dentro de los animales de compañía, los canes son los que se encuentran con mayor frecuencia en los hogares de Lima y probablemente en diferentes regiones del Perú y Latinoamérica (Llalla, 2012). Los canes cumplen una serie de actividades, entre ellas la de guardianes, cazadores o simplemente animales de compañía, cuya función principal es la de convivir con el hombre (Álvarez y Domínguez, 2001). Estos animales también han sido utilizados para promover la salud a través de programas de terapia asistida donde los canes ayudan a los pacientes, especialmente en las áreas de rehabilitación física y recuperación sicológica y emocional (Correa et al., 2011).

El comportamiento de la población de canes varía según las diferentes regiones, principalmente por el estado de ingresos locales y el tipo de residencia humana (Serafini et al., 2008). La tenencia de estos animales implica la responsabilidad de los propietarios de contar con un presupuesto para sus necesidades básicas, espacio, tiempo y el respeto de las normas establecidas por las autoridades sanitarias, asegurando así su bienestar, cuidado y supervivencia (ANPA, 2004; Espinola, 2004). Sin embargo, la tenencia no responsable de canes representa un problema para la salud pública, la que se manifiesta en un aumento en el riesgo de transmisión de enfermedades zoonóticas, contaminación 
ambiental con heces y accidentes por mordedura en las personas (León et al., 2013).

Debido a la implicancia de esta problemática sobre la salud pública, el año 2001 se aprobó la Ley N. 27596 que regula el «Régimen Jurídico de Canes» y el 2012 se aprobó su reglamento. Las Municipalidades distritales y provinciales se encuentran obligadas a hacerla efectiva a través de la elaboración y promulgación de ordenanzas municipales en busca de fomentar una tenencia responsable de los canes. En ese contexto, el objetivo del estudio fue evaluar el conocimiento y prácticas de la población asociadas al cumplimiento de las ordenanzas emitidas por las municipalidades distritales de Lima dentro del marco de la aplicación de la Ley N. ${ }^{\circ}$ 27596.

\section{Materiales y Métodos}

El estudio se realizó en los siguientes distritos de Lima, Perú: La Molina, Magdalena, Miraflores, San Borja, San Isidro, San Miguel, Surco (estrato socioeconómico alto), Ate, Breña, Chorrillos, Jesús María, La Victoria, Lima, Los Olivos, San Martín de Porras (estrato socioeconómico medio) y Carabayllo, Comas, Puente Piedra, San Juan de Lurigancho, Villa el Salvador (estrato socioeconómico bajo). Los distritos fueron calificados a los estratos socioeconómicos correspondientes teniendo en consideración los planos estratificados de Lima Metropolitana según el Instituto Nacional de Estadística e Informática (INEI, 2016). El diseño del estudio correspondió a una investigación observacional, transversal y descriptiva.

El tamaño de muestra fue determinado mediante la fórmula de comprobación de una proporción para poblaciones grandes. Se utilizó un nivel de confianza del 99\%, un error máximo admisible del $5 \%$ y una proporción referencial para conocimientos de la Ley $\mathrm{N} .^{\circ}$ 27596 del 50\% (valor utilizado cuando se desconoce una proporción referencial previa).
El tamaño de muestra determinado fue de 664 encuestas como mínimo. Los encuestados fueron dueños de animales de compañía, mayores de edad y residentes de los distritos involucrados en el estudio.

El instrumento de recolección de información incluyó las siguientes variables: a) número de personas y de canes que habitan las viviendas, b) Conocimiento acerca de los principales aspectos normativos incluidos en la Ley N. ${ }^{\circ} 27596$ y de la existencia de una ordenanza municipal al respecto en el distrito, c) prácticas asociadas a la Ley N. ${ }^{\circ} 27596$ en el manejo de los canes. El instrumento fue sometido a una evaluación por juicio de expertos y la versión final se obtuvo cuando los evaluadores aceptaron la última versión del instrumento.

La aplicación del instrumento se realizó en personas que se encontraban en espacios públicos de los distritos seleccionados, a quienes se solicitó la participación voluntaria en el estudio. Las preguntas fueron realizadas por el encuestador quien anotó las respuestas en la hoja de encuesta y la entrevista tuvo una duración promedio de 15 minutos. La información obtenida en las encuestas fue transferida a una hoja de cálculo en el programa Microsoft Excel y la información fue analizada mediante el programa estadístico STATA 15.0 utilizando estadística descriptiva. Los resultados se resumieron en tablas de frecuencia. La asociación entre las variables dependientes (conocimientos y prácticas) con la variable independiente (estrato socioeconómico) fue evaluada mediante la prueba de Chi cuadrado. Los resultados generales para conocimientos y prácticas se presentaron en proporciones con sus respectivos intervalos de confianza.

La participación en el estudio fue voluntaria y su aceptación implicó la firma de un consentimiento informado. Asimismo, el estudio contó con la aprobación del Comité Institucional de Ética de la Universidad Peruana Cayetano Heredia (Lima, Perú). 
Resultados

El estudio recolectó 783 encuestas de los cuales $35.5 \%$ (278) correspondió a personas del sexo masculino y $64.5 \%$ (505) al sexo femenino. La edad media de los encuestados fue de 31.1 años con una desviación estándar de 12.6 años (valores extremos mínimo de 18 años y máximo de 85 años). El 37.0\% (290) correspondió al estrato socioeconómico alto, $32.6 \%$ (255) al estrato medio y $30.4 \%$ (238) al estrato bajo. El promedio de canes por vivienda fue de 1.7 (1294/ 782). La media según estratos socioeconómicos tuvo una relación inversa, siendo de $1.5(447 / 290), 1.6(413 / 255)$ y $1.8(434 / 238)$ para los estratos socioeconómicos alto, medio y bajo, respectivamente.

Del total de encuestados, 67.6\% (529) mencionaron conocer que existían razas potencialmente peligrosas, correspondiendo a $65.2 \%$ (189), 68.2\% (174) y 69.7\% (166) para los estratos alto, medio y bajo, respectivamente. La distribución de la frecuencia de razas de canes reconocidos como peligrosos se presentan en el Cuadro 1. Así mismo, 24.9\% (195) de los encuestados mencionaron conocer que existen requisitos para ser propietario de un can de raza potencialmente peligroso, correspondiendo a $28.6 \%$ (83), $23.1 \%$ (59) y $22.3 \%$ (53) para los estratos alto, medio y bajo, respectivamente $(\mathrm{p}<0.001)$.

El 35.1\% (275) de los encuestados mencionaron conocer que la castración era un método para disminuir la agresividad de los canes, siendo las respuestas de $36.2 \%$ (105), $32.5 \%$ (83) y $36.6 \%$ (87) en los estratos socioeconómicos alto, medio y bajo, respectivamente. Sin embargo, la práctica de esterilización y castración de los canes fue reconocida en forma limitada en los encuestados. Solamente 29.1\% (228) habría decidido controlar la reproducción en forma quirúrgica, con diferencias significativas por estrato (36.2\% (105) en el alto, 21.2\% (54) en el medio y $29.0 \%$ (69) en el bajo $[p<0.001])$.
El 24.8\% (194) de los encuestados opinaron que los canes deben de ser adiestrados en centros específicos para dicho fin, con diferencias de opinión según el estrato socioeconómico (30.7\% [89], 20.4\% [52] y $22.3 \%$ [53] para los estratos alto, medio y bajo, respectivamente $)(\mathrm{p}=0.011)$.

En cuanto al requerimiento de autorización del SENASA para movilización de canes con el extranjero (entrada o salida del país), $65.8 \%$ (515) de los encuestados opinaron que era un requisito (78.6\% [228], 60.8\% [153] y $56.3 \%$ [134] para los estratos socioeconómicos alto, medio y bajo, respectivamente) $(p=0.000)$. En el caso de ese requerimiento para la movilización dentro del país, 23.9\% (187) indicó correctamente que no se requería $(29.7 \%$ [86], 20.4\% [52] y $20.6 \%$ [49] para la condición de alto, medio y bajo, respectivamente) $(\mathrm{p}<0.020)$. En cuanto a la práctica, $26.7 \%$ (209) de los encuestados señaló haber realizado viajes interprovinciales acompañado por su can, habiendo respuestas similares entre los tres estratos, habiendo hecho uso de trasporte privado en el $74.1 \%$ (60), $47.6 \%$ (30) y $33.8 \%$ (22) por los encuestados de los estratos alto, medio y bajo, respectivamente $(\mathrm{p}<0.001)$.

En el caso de quién debe asumir la responsabilidad de los gastos de atención médica en caso de accidentes por mordedura, la respuesta mayoritaria para todos los estratos socioeconómicos fue que debe ser el dueño del can, aunque con una ligera menor proporción en los encuestados del estrato más bajo (Cuadro 2). El 12.8\% (100) de los encuestados señaló que sus canes mordieron en alguna ocasión a una persona (7.6\% [22], $11.8 \%$ [30] y $20.2 \%$ [48] en los niveles alto, medio y bajo, respectivamente) $(\mathrm{p}<0.001)$, donde el $72 \%$ de los propietarios reconocieron el gasto médico (81.8\% [18] en el alto, $73.3 \%$ [(22] en el medio y $66.7 \%$ [(32] en el bajo) $(\mathrm{p}<0.020)$. 
Cuadro 1. Razas de canes consideradas como potencialmente peligrosas por personas entrevistadas ${ }^{1}$ en 20 distritos de Lima, Perú (2018)

\begin{tabular}{|c|c|c|c|c|c|c|c|c|}
\hline \multirow{3}{*}{$\begin{array}{l}\text { Razas de canes } \\
\text { potencialmente peligrosos }\end{array}$} & \multicolumn{8}{|c|}{ Estrato socioeconómico } \\
\hline & \multicolumn{2}{|c|}{$\begin{array}{c}\text { Alto } \\
(\mathrm{n}=189)\end{array}$} & \multicolumn{2}{|c|}{$\begin{array}{l}\text { Mediano } \\
(\mathrm{n}=174)\end{array}$} & \multicolumn{2}{|c|}{$\begin{array}{c}\text { Bajo } \\
(n=166)\end{array}$} & \multicolumn{2}{|c|}{$\begin{array}{c}\text { Total } \\
(n=529)\end{array}$} \\
\hline & $\mathrm{n}$ & $\%$ & $\mathrm{n}$ & $\%$ & $\mathrm{n}$ & $\%$ & $\mathrm{n}$ & $\%$ \\
\hline \multicolumn{9}{|c|}{ Según la Resolución Ministerial 1776-2002-SA/DM } \\
\hline Pitbull & 165 & 87.3 & 152 & 87.4 & 140 & 84.3 & 457 & 86.4 \\
\hline Rottweiler & 68 & 36.0 & 76 & 43.7 & 67 & 40.4 & 211 & 39.9 \\
\hline Doberman & 16 & 8.5 & 23 & 13.2 & 22 & 13.3 & 61 & 11.5 \\
\hline Dogo Argentino & 8 & 4.2 & 6 & 3.4 & 1 & 0.6 & 15 & 2.8 \\
\hline Fila Brasilero & 2 & 1.1 &.-- &.-- &.-- &.-- & 2 & 0.4 \\
\hline \multicolumn{9}{|c|}{ Otras razas mencionadas en la encuesta } \\
\hline Bóxer & 12 & 6.3 & 11 & 6.3 & 10 & 6.0 & 33 & 6.2 \\
\hline Shih Tzu & 12 & 6.3 & 11 & 6.3 & 10 & 6.0 & 33 & 6.2 \\
\hline Pastor Alemán & 2 & 1.1 & 2 & 1.1 & 4 & 2.4 & 8 & 1.5 \\
\hline Bull Terrier & 4 & 2.1 & 8 & 4.6 & 3 & 1.8 & 15 & 2.8 \\
\hline AmBulTer & 1 & 0.5 &.-- &.-- &.-- &.-- & 1 & 0.2 \\
\hline American Stanford & 1 & 0.5 &.-- &.-- &.-- &.-- & 1 & 0.2 \\
\hline Bulldog & 1 & 0.5 &.-- &.-- &.-- &.-- & 1 & 0.2 \\
\hline Cruzados & 1 & 0.5 &.-- &.-- &.-- &.-- & 1 & 0.2 \\
\hline Pastor Belga & 1 & 0.5 &.-- &.-- &.-- &.-- & 1 & 0.2 \\
\hline Chihuahua &.-- &.-- & 1 & 0.6 & 2 & 1.2 & 3 & 0.6 \\
\hline Labrador &.-- &.-- & 2 & 1.1 &.-- &.-- & 2 & 0.4 \\
\hline Mastín Napolitano &.-- &.-- & 2 & 1.1 &.-- &.-- & 2 & 0.4 \\
\hline Pekinés &.-- &.-- & 1 & 0.6 &.-- &.-- & 1 & 0.2 \\
\hline
\end{tabular}

${ }^{1}$ Los encuestados pudieron ofrecer más de una respuesta

El 27.5\% (215) mencionó conocer el tiempo que un can mordedor debe de permanecer en observación para el control de la rabia, aunque solo $29.8 \%$ (64) de ellos ofreció la respuesta correcta $(8.2 \%$ respecto al total de encuestados) (Cuadro 3). De otra parte, $87.2 \%$ (783) de los encuestados mencionaron conocer que existe enfermedades transmitidas por la mordedura de un can; de ello, 98.5\% (673) respondió que era la rabia
(86.0\% de respuestas acertadas respecto al total de encuestados). El detalle de estas respuestas por estrato socioeconómico se presenta en el Cuadro 4.

La obligatoriedad que tienen los tenedores de canes potencialmente peligrosos de adquirir un seguro contra los daños que sus animales pudieran ocasionar era conocido solamente por el $29.4 \%$ (230) de los 
Cuadro 2. Opinión de las personas encuestadas en 20 distritos de Lima respecto al encargado de asumir los gastos de atención médica en caso de accidentes por mordedura (2018)

\begin{tabular}{lcccccccc}
\hline & \multicolumn{7}{c}{ Estrato socioeconómico } \\
\cline { 2 - 9 } $\begin{array}{l}\text { ¿Quién asume la } \\
\text { responsabilidad? }\end{array}$ & $\begin{array}{c}\text { Alto } \\
(\mathrm{n}=290)\end{array}$ & $\begin{array}{c}\text { Mediano } \\
(\mathrm{n}=255)\end{array}$ & $\begin{array}{c}\text { Bajo } \\
(\mathrm{n}=238)\end{array}$ & $\begin{array}{c}\text { Total } \\
(\mathrm{n}=783)\end{array}$ \\
\cline { 2 - 10 } & $\mathrm{n}$ & $\%$ & $\mathrm{n}$ & $\%$ & $\mathrm{n}$ & $\%$ & $\mathrm{n}$ & $\%$ \\
\hline Dueño del can & 283 & 97.6 & 247 & 96.9 & 214 & 89.9 & 744 & 95.0 \\
Persona afectada & 5 & 1.7 & 1 & 0.4 & 8 & 3.4 & 14 & 1.8 \\
Municipalidad & 1 & 0.3 & 1 & 0.4 & 1 & 0.4 & 3 & 0.4 \\
Centro de Salud & 1 & 0.3 & 4 & 1.6 & 12 & 5.0 & 17 & 2.2 \\
No sabe / no opina &.-- &.-- & 2 & $0-8$ & 3 & 1.2 & 5 & 0.7 \\
\hline
\end{tabular}

Cuadro 3. Conocimiento de las personas encuestadas en 20 distritos de Lima respecto al tiempo de observación en internamiento de un can mordedor (2018)

\begin{tabular}{lcccccccc}
\hline & \multicolumn{7}{c}{ Estrato socioeconómico } \\
\cline { 2 - 10 } $\begin{array}{l}\text { Tiempo de observación del } \\
\text { can mordedor }\end{array}$ & $\begin{array}{c}\text { Alto } \\
(\mathrm{n}=290)\end{array}$ & \multicolumn{2}{c}{$\begin{array}{c}\text { Mediano } \\
(\mathrm{n}=255)\end{array}$} & \multicolumn{2}{c}{$\begin{array}{c}\text { Bajo } \\
(\mathrm{n}=238)\end{array}$} & \multicolumn{2}{c}{$\begin{array}{c}\text { Total } \\
(\mathrm{n}=783)\end{array}$} \\
\cline { 2 - 10 } & $\mathrm{n}$ & $\%$ & $\mathrm{n}$ & $\%$ & $\mathrm{n}$ & $\%$ & $\mathrm{n}$ & $\%$ \\
\hline $\begin{array}{l}\text { Total que afirmaron } \\
\text { conocer }\end{array}$ & 89 & 30.7 & 54 & 21.2 & 72 & 30.3 & 215 & 27.5 \\
$\begin{array}{l}\text { Respuesta correcta en los } \\
\text { que contestaron }\end{array}$ & 31 & 34.8 & 18 & 33.3 & 15 & 20.8 & 64 & 29.8 \\
$\begin{array}{l}\text { Respuesta correcta } \\
\text { respecto al total }\end{array}$ & 31 & 10.7 & 18 & 7.1 & 15 & 6.3 & 64 & 8.2 \\
\hline
\end{tabular}

Cuadro 4. Conocimiento de las personas encuestadas en 20 distritos de Lima acerca de la enfermedad transmitida a través de la mordedura de un can (2018)

\begin{tabular}{lccccccccc}
\hline & \multicolumn{7}{c}{ Estrato socioeconómico } \\
\cline { 2 - 10 } $\begin{array}{l}\text { Enfermedad transmisible } \\
\text { por mordedura del can }\end{array}$ & $\begin{array}{c}\text { Alto } \\
(\mathrm{n}=290)\end{array}$ & \multicolumn{2}{c}{$\begin{array}{c}\text { Mediano } \\
(\mathrm{n}=255)\end{array}$} & \multicolumn{2}{c}{$\begin{array}{c}\text { Bajo } \\
(\mathrm{n}=238)\end{array}$} & \multicolumn{2}{c}{$\begin{array}{c}\text { Total } \\
(\mathrm{n}=783)\end{array}$} \\
\cline { 2 - 10 } & $\mathrm{n}$ & $\%$ & $\mathrm{n}$ & $\%$ & $\mathrm{n}$ & $\%$ & $\mathrm{n}$ & $\%$ \\
\hline $\begin{array}{l}\text { Total que afirmaron } \\
\text { conocer }\end{array}$ & 265 & 91.4 & 216 & 84.7 & 202 & 84.9 & 783 & 87.2 \\
$\begin{array}{l}\text { Respuesta correcta en los } \\
\text { que contestaron }\end{array}$ & 262 & 98.9 & 212 & 98.1 & 199 & 98.5 & 673 & 98.5 \\
$\begin{array}{l}\text { Respuesta correcta } \\
\text { respecto al total }\end{array}$ & 262 & 90.3 & 212 & 83.1 & 199 & 83.6 & 673 & 86.0 \\
\hline
\end{tabular}


Cuadro 5. Métodos de identificación de canes utilizados por los propietarios de canes encuestados en 20 distritos de Lima (2018)

\begin{tabular}{lcccccccc}
\hline & \multicolumn{7}{c}{ Estrato socioeconómico } \\
\cline { 2 - 9 } Identificación del can & $\begin{array}{c}\text { Alto } \\
(\mathrm{n}=207)\end{array}$ & \multicolumn{2}{c}{$\begin{array}{c}\text { Mediano } \\
(\mathrm{n}=98)\end{array}$} & $\begin{array}{c}\text { Bajo } \\
(\mathrm{n}=73)\end{array}$ & $\begin{array}{c}\text { Total } \\
(\mathrm{n}=378)\end{array}$ \\
\cline { 2 - 10 } & $\mathrm{n}$ & $\%$ & $\mathrm{n}$ & $\%$ & $\mathrm{n}$ & $\%$ & $\mathrm{n}$ & $\%$ \\
\hline Collar con placa & 175 & 84.5 & 82 & 83.7 & 60 & 82.2 & 317 & 83.9 \\
Microchip & 21 & 10.2 & 3 & 3.1 & 3 & 4.1 & 27 & 7.1 \\
Otros (tatuajes, tarjetas) & 11 & 5.3 & 13 & 13.2 & 10 & 13.7 & 34 & 9 \\
\hline
\end{tabular}

encuestados (32.8\% [95], 35.3\% [90] y $18.9 \%$ [45] para los estratos socioeconómicos alto, medio y bajo, respectivamente) $(\mathrm{p}<0.001)$. La obligatoriedad de que la Policía Nacional auxilie a las personas víctimas de accidentes por mordedura de canes y que realice las investigaciones correspondientes fue reconocida por el $58.7 \%$ (460) de los encuestados (67.9\% [197], 55.3\% [141] y $51.3 \%$ [122]) para los estratos alto, medio y bajo, respectivamente) $(\mathrm{p}<0.001)$.

El 96.3\% (754) de los encuestados señala que el responsable de recoger las heces de los canes recae sobre los dueños $(96.6 \%$ [280] según el estrato socioeconómico alto, 97.3\% [248] en el medio y $95.0 \%$ [226] en el bajo). En cuanto a la práctica de recojo de heces de los canes por parte de los propietarios se encontró una proporción general de $82.6 \%$ (647), siendo de $92.8 \%$ (269) en el estrato alto, $81.2 \%$ (207) en el medio y de $71.8 \%$ en el bajo $(171)(\mathrm{p}<0.001)$.

El 51.7\% (405) del total de los encuestados afirmaron no poseer un sistema de identificación de los animales, donde esta respuesta alcanzó en el 69.3\% (165) de los encuestados del estrato socioeconómico bajo, seguido del medio con $61.6 \%$ (157) y el alto con $28.6 \%(83)(\mathrm{p}<0.001)$. El tipo de identificación preferente fue el collar y la proporción de los dueños de canes que lo utilizaron seguía una relación directa con el estrato de donde provenían (Cuadro 5). La práctica de registro de canes en la municipalidad alcanzó un $17.8 \%$ (139) entre en total de encuestados (32.1\% [93], 12.5\% [32] y 5.9\% [14] para los estratos alto, medio y bajo, respectivamente) $(\mathrm{p}<0.001)$.

La práctica de pasear a los canes fue mayoritaria. En general, 91.2\% (714) mencionó hacerlo, alcanzando proporciones de $94.8 \%$ (275), $89.4 \%$ (228) y $88.7 \%$ (211) para los estratos socioeconómicos alto, medio y bajo, respectivamente $(\mathrm{p}<0.05)$; no obstante, la forma como se paseaba al can variaba de acuerdo a la procedencia de los encuestados $(\mathrm{p}<0.001)$ (Cuadro 6)

A la interrogante acerca de haber recibido alguna charla de tenencia responsable de animales de compañía, solo 14.3\% (112) del total de encuestados respondió afirmativamente (15.5\% [45] en el alto, $14.9 \%$ [38] en el medio y $12.2 \%$ [29] en el bajo). Los medios por los que recibieron la información fueron muy variados, siendo estos de tipo autodidacta, establecimiento de salud, insti- 
Cuadro 6. Forma de paseo de los canes entre los propietarios de canes encuestados en 20 distritos de Lima (2018)

\begin{tabular}{lcccccccc}
\hline & \multicolumn{7}{c}{ Estrato socioeconómico } \\
\cline { 2 - 9 } Forma de paseo & $\begin{array}{c}\text { Alto } \\
(\mathrm{n}=275)\end{array}$ & $\begin{array}{c}\text { Mediano } \\
(\mathrm{n}=228)\end{array}$ & $\begin{array}{c}\text { Bajo } \\
(\mathrm{n}=211)\end{array}$ & $\begin{array}{c}\text { Total } \\
(\mathrm{n}=714)\end{array}$ \\
\cline { 2 - 9 } & $\mathrm{n}$ & $\%$ & $\mathrm{n}$ & $\%$ & $\mathrm{n}$ & $\%$ & $\mathrm{n}$ & $\%$ \\
\hline Con correa & 239 & 86.9 & 188 & 82.5 & 146 & 69.2 & 573 & 80.3 \\
Con bozal & 2 & 0.7 & 0 & 0.0 & 1 & 0.5 & 3 & 0.4 \\
Con correa y bozal & 9 & 3.3 & 8 & 3.5 & 4 & 1.9 & 21 & 2.9 \\
Suelto & 25 & 9.1 & 32 & 14.0 & 60 & 28,4 & 117 & 16.4 \\
\hline
\end{tabular}

Cuadro 7. Responsable de hacer cumplir la Ley que regula el Régimen Jurídico de Canes según opinión de personas encuestadas en 20 distritos de Lima (2018)

\begin{tabular}{lcccccccc}
\hline & \multicolumn{7}{c}{ Estrato socioeconómico } \\
\cline { 2 - 9 } Responsable & $\begin{array}{c}\text { Alto } \\
(\mathrm{n}=290)\end{array}$ & \multicolumn{2}{c}{$\begin{array}{c}\text { Mediano } \\
(\mathrm{n}=255)\end{array}$} & $\begin{array}{c}\text { Bajo } \\
(\mathrm{n}=238)\end{array}$ & $\begin{array}{c}\text { Total } \\
(\mathrm{n}=783)\end{array}$ \\
\cline { 2 - 10 } & $\mathrm{n}$ & $\%$ & $\mathrm{n}$ & $\%$ & $\mathrm{n}$ & $\%$ & $\mathrm{n}$ & $\%$ \\
\hline Municipalidad & 141 & 48.6 & 68 & 26.7 & 113 & 47.5 & 322 & 41.1 \\
Ministerio de Salud & 49 & 16.9 & 34 & 13.3 & 29 & 12.2 & 112 & 14.3 \\
Otros & 10 & 3.4 & 5 & 2.0 & 7 & 2.9 & 22 & 2.8 \\
No sabe / No opina & 90 & 31.0 & 148 & 58.0 & 89 & 37.4 & 327 & 41.8 \\
\hline
\end{tabular}

tución educativa, entrenador, expropietaria, familiar, médico veterinario, municipalidad, ONG, universidad, servicio veterinario (clínica o consultorio), sin encontrarse predominancia en alguno de ellos.

Respecto a haber escuchado acerca de la existencia de la Ley N. ${ }^{\circ} 27596$ «Ley que Regula el Régimen Jurídico de Canes», $16.2 \%$ (127) del total respondió afirmativamente $(18.3 \%$ [53] en el estrato socioeconómico alto, $13.7 \%$ [35] en el medio y
$16.4 \%$ [39] en el bajo). El 41.1\% (322) del total de encuestados indicó que la entidad responsable de hacer cumplir dicha Ley eran las municipalidades y el 14.3\% (112) señaló al Ministerio de Salud (Cuadro 7). El conocimiento respecto a si la municipalidad de su distrito poseía una Ordenanza Municipal con relación a la Ley $\mathrm{N} .^{\circ} .27596$ fue bajo $(8.4 \%)$, siendo de $9.6 \%$ (17) en estrato socioeconómico alto, $9.1 \%$ (8) en el medio y $6.3 \%$ (8) en el bajo. 


\section{Discusión}

El estudio recogió opinión de poblaciones de diferentes distritos y consecuentemente de diferentes estratos socioeconómicos a fin de obtener información representativa de Lima Metropolitana. El agrupamiento de las zonas de muestreo y distritos se realizó con la finalidad de segmentar los grupos o estratos socioeconómicos a fin de que se pueda tener información de la variación de los conocimientos y prácticas de acuerdo con esta variable. Si bien es cierto en cada distrito se puede encontrar más de un estrato socioeconómico representado, las entrevistas se aplicaron al segmento de la población correspondiente al estrato socioeconómico en el cual fue agrupado el distrito. Las variadas características demográficas de los participantes han permitido obtener información que facilita la extrapolación de los resultados a la población general

El promedio de canes por vivienda tuvo una tendencia inversa con relación al estrato socioeconómico en estudio. Aunque no se encontró diferencia estadística entre las medias, la tendencia de los resultados concuerda con el estudio de Ibarra et al. (2003) en Chile, quien reporta que en las comunas que históricamente se encuentran mayormente las personas con niveles de ingreso económico más alto se asocian a una menor densidad poblacional de canes.

Más de la mitad de los encuestados reconocieron saber que existen razas de canes potencialmente peligrosas. De ellas, la raza más reconocida como tal fue el Pit Bull Terrier. La normativa peruana ya define a un conjunto de razas como potencialmente peligrosas siendo estas el Pit Bull Terrier, Dogo Argentino, Fila Brasilero, Tosa Japonesa, Bul Mastiff, Doberman y Rotweller (Resolución Ministerial N. ${ }^{\circ}$ 1776-2002-SA/DM). El reconocer al Pit Bull Terrier en primer lugar y después al Rotweiler y Doberman como potencialmente peligrosos se puede deber a que perros de estas razas se han involucrado en una serie de casos de accidentes por mordedura, tomando más notoriedad cuando estas noticias aparecen en reportes nacionales e internacionales asociados a casos fatales.

Por otro lado, se debe de tener en cuenta que una proporción importante de los encuestados, propietarios de canes, desconocían sobre la existencia de razas de canes consideradas potencialmente peligrosas. A esto se suma el hecho de que las razas como Tosa Japonés, Fila Brasilero, y Dogo Argentino fueron razas pobremente reconocidas debido probablemente a que sus características no has sido debidamente difundidas o porque su número es bajo con relación a las otras razas grandes. Ambas situaciones representan un riesgo, porque de ser propietario de alguno de ellos o persona que entre en contacto con estos canes, puede que no tomen las medidas de manejo que exige la norma a fin de prevenir algún tipo de accidente.

La existencia de requisitos para ser propietario de canes potencialmente peligrosos es conocida por una proporción baja de entrevistados, por lo que se requiere que se difundan con mayor énfasis. Ello debe de estar incluido en las ordenanzas municipales emitidas con relación al cumplimiento de la Ley N. ${ }^{\circ} 27596$ y se debe velar por su cumplimiento, de tal forma que cuando los dueños se encuentren involucrados en accidentes que afecten la salud e integridad de las personas, puedan ser localizados y obligados a asumir las consecuencias de este.

Si bien el uso de la castración como estrategia para el control de la agresividad de los canes machos fue reconocida por uno de cada tres personas, su aceptación como práctica rutinaria es baja. Rojas et al. (2019) reportan que solo el $10 \%$ de los canes que son sometidos a control reproductivo quirúrgico son machos, demostrando que la aceptación cultural de la castración es baja.

El desarrollo de actividades de adiestramiento de canes debe realizarse en centros habilitados especialmente para estos fi- 
nes y con las seguridades necesarias para el resguardo de la seguridad e integridad de las personas (Ley N. ${ }^{\circ} 27596$, Cap., 2 Art. 7); sin embargo, muchos de los encuestados desconocen esta exigencia de la norma por lo que serían pasibles a multas al exponer a terceros a accidentes cuando estas prácticas se realizan clandestinamente en lugares sin equipos niherramientas necesarias para este fin.

El requerimiento de la autorización del SENASA para trasladar canes hacia el extranjero y viceversa ha de ser importante para la movilización sanitaria de los animales y de esta manera evitar el traslado de enfermedades exóticas entre países. El movimiento de animales a nivel del interior del país no está regulado y en la actualidad se han ofrecido mayores facilidades para el traslado en transporte público (ómnibus interprovinciales y aviones) y particular; práctica que habría realizado alrededor del $25 \%$ de los encuestados. Es importante que los propietarios asuman con responsabilidad estas facilidades, especialmente si se considera que se tiene áreas endémicas para zoonosis como es la presencia de rabia en algunas regiones del Perú (Puno y Arequipa) (Recuenco, 2019). Esto hace necesario reevaluar la necesidad de regular este movimiento como herramienta para la vigilancia epidemiológica y la trazabilidad en caso de brotes de enfermedades de importancia en salud pública.

La responsabilidad de asumir los costos de los accidentes por mordedura que ocasione el can de su propiedad es conocida por la mayoría de los encuestados, lo cual, según el artículo 14 de la Ley N. ${ }^{\circ} 27596$, si un can ocasiona una lesión grave a una persona, el dueño del can está obligado a asumir el costo total de la hospitalización, medicamentos y cirugía reconstructiva necesaria hasta su recuperación total, sin perjuicio de la indemnización por daños y perjuicios a que hubiere lugar. La mayor proporción de propietarios que asumieron la responsabilidad de los daños producido por sus canes guardó una relación directa con las condiciones socioeconómicas del distrito donde residían los encuestados. Es importante tomar en consideración que la reparación que debe asumir el propietario del can no solo está relacionado a la lesión producida por la mordedura, sino que también a las intervenciones quirúrgicas para superar las secuelas estéticas en los accidentados cuando las mordeduras son graves (Romero et al., 2014).

La proporción de encuestados que dice conocer el tiempo que se debe de observar a un can agresor después de una mordedura fue baja, y más aún la proporción que respondió correctamente a la pregunta. Ello requiere de una mayor difusión especialmente en zonas no endémicas de la rabia (Talavera et al., 2014). Además, la observación del animal agresor durante 10 días y el resultado de esta observación tienen que considerarse antes de determinar si es necesaria el inicio o culminación de un programa de vacunación antirrábica (MINSA, 2017). Robles et al. (2017) mencionan que las regulaciones acerca de la responsabilidad de los dueños respecto a daños que causen los canes a terceros es mayoritaria en las Ordenanzas Municipales de los distritos de Lima (79.3\%), pero la vigilancia o internamiento del can mordedor por 10 días en centro antirrábico o establecimiento de salud se menciona con menor frecuencia $(48.3 \%)$.

La exigencia de adquirir un seguro de responsabilidad civil en caso de daños a terceros ocasionados por canes potencialmente peligrosos es conocida en mayor proporción en personas de estrato socioeconómico alto, en comparación a los de estrato bajo. Ello es necesario que sea conocido por la población, especialmente la de más bajos recursos, quienes suelen sufrir por la falta de recursos para atender a los accidentados. Ello debe de estar acompañado por el conocimiento de la obligatoriedad que tiene la Policía Nacional del Perú de auxiliar a los accidentados y velar por el cumplimiento de la Ley N. ${ }^{\circ} 27596$.

En el tema del recojo de las heces, el conocimiento de la obligatoriedad y la práctica por parte de los propietarios fue alto, lo 
que es importante para evitar la contaminación del ambiente, producto de la costumbre de las personas de llevar a sus canes a defecar a ambientes públicos. Su cumplimiento se favorece si se promueve el acondicionamiento de tachos para la disposición de las heces que se recogen.

La identificación de los canes tuvo una relación inversa a los estratos socioeconómicos. La mayoría de encuestados que identificaban a sus animales utilizaban métodos diferentes al microchip, los cuales no permiten reconocer al dueño en caso de una falta cometida por el animal o cuando este se extravíe. El uso del microchip es aún limitado debido a su relativo alto costo. Jara (2009) encontró que los médicos veterinarios encuestados en Chile indicaron que el mejor método de identificación a nivel masivo lo constituiría el microchip, principalmente por la inviolabilidad de dicha identificación, pero en contraste, los dirigentes comunitarios encuestados preferían la placa de identificación, principalmente por su bajo costo.

El registro de canes en la municipalidad es una de las practicas menos comunes entre los encuestados, más allá que la mayoría de las ordenanzas municipales la incluye. Es por esto que ninguna municipalidad puede aseverar que conoce la población de canes existentes en su distrito. Esta información es importante para la salud pública ya que a partir de ella se puede realizar una adecuada planificación y evaluación de las coberturas de las campañas de vacunación antirrábica (León et al., 2014). Además, estos registros, junto con la identificación de los canes, permitirían identificar a los propietarios de canes mantenidos en condición de vagabundos o que se encuentren involucrados en accidentes por mordedura o daño a terceros.

La Ley N. ${ }^{\circ} 27596$ menciona que se debe conducir a los canes por lugares públicos con correas cuya extensión y resistencia sean suficientes para asegurar el control sobre ellos. En el caso de canes considerados potencialmente peligrosos deben conducirse adicionalmente con bozal. La conducción debe realizarla el propietario o cualquier otra persona adulta con capacidad física y mental para ejercer el control adecuado sobre el animal (Ley N. ${ }^{\circ} 27596$, Cap. 2, Art 5). El incumplimiento de esta parte de la norma puede exponer a terceros a zoonosis parasitarias cuando los animales que defecan en ambientes públicos no se encuentren desparasitados (Leguía, 2002).

La proporción de encuestados que afirman haber recibido capacitación en temas de tenencia responsable de animales de compañía es baja. En este sentido, se debe de tener en cuenta que el Reglamento de la Ley N. ${ }^{\circ}$ 27596 establece que el Ministerio de Salud, Municipalidades y organizaciones reconocidas por el Estado, en coordinación con el Ministerio de Educación, desarrollarán programas de capacitación y educación sanitaria sobre la tenencia responsable de canes, zoonosis, sus mecanismos de transmisión, medidas sanitarias y formas de prevención y protección de la salud pública. La coordinación de las municipalidades y el sector educación ha de resultar valiosa debido a que por medio de la escuela se puede hacer llegar información a gran cantidad de escolares al incorporar los temas de tenencia responsable de animales de compañía entre las temáticas que se les ofrecen en los cursos regulares, e inclusive hacer llegar esa información a los adultos a través de las denominadas escuelas de padres que suelen realizar las instituciones educativas.

Los entrevistados en general, independientemente del estrato socioeconómico, poseían escaso conocimiento acerca de la Ley N. ${ }^{\circ} 27596$ y no reconocen a las municipalidades como responsable de hacerla cumplir. En ese sentido, Robles et al. (2017) mencionaron que es importante que las municipalidades, además de aprobar las ordenanzas que permitan que viabilice la aplicación de esta ley, se encargue de difundir los aspectos más resaltantes de la misma. Esto se puede realizar mediante charlas, talleres o presentaciones en escuelas, organizaciones vecinales 
(comedor popular, vasos de leche, asociaciones de viviendas, etc.) y otros medios de difusión (volantes, perifoneo, etc.) para informar y concientizar a la población, en especial a los dueños de animales de compañía sobre la importancia de respetar y cuidarlos, así como las obligaciones que tienen como propietarios.

\section{Conclusiones}

El estudio concluye que el conocimiento y las practicas relacionadas al cumplimiento de la Ley N. ${ }^{\circ} 27596$, Ley que Regula el Régimen Jurídico de Canes es escasa en los distritos de Lima Metropolitana. Sin embargo, dentro de lo poco que se conoce y se practi$\mathrm{ca}$, existe una tendencia a que se cumpla en los sectores de mejor condición socioeconómica.

\section{Literatura Citada}

1. Álvarez E, Domínguez, J. 2001. Programa para el control integral de la población canina. AMMVEPE 12: 83-91.

2. [ANPA] Asociación Nacional Protectora de Animales. 2004. Manual básico de tenencia responsable de perros y gatos. Costa Rica: ANPA. 23 p.

3. Correa J, Davis M, Ruffin W, Ebert R, Floyd J. 2011. La compañía del perro y sus beneficios para el ser humano. USA: Alabama A\&M and Auburn Universities. 9 p.

4. Decreto Supremo $.^{\circ} 006-2002-S A$ que aprueba el Reglamento de la Ley Nro. 27596, Ley que regula el Régimen Jurídico de Canes. [Internet]. Disponible en: ftp://ftp2.minsa.gob.pe/normaslegales/2002/DS006-2002.pdf

5. Espinola F. 2004. Estimación de la población canina callejera y supervisada en las calles de la ciudad de Santiago, Región Metropolitana. Tesis de Médico Veterinario. Santiago, Chile: Univ. de Chile. $53 \mathrm{p}$.
6. Ibarra L, Morales M, Acuña P. 2003. Aspectos demográficos de la población de perros y gatos en la ciudad de Santiago, Chile. Avances Cienc Vet 18: 13-20.

7. [INEI] Instituto Nacional de Estadística e Informática. 2016. Planos estratificados de Lima Metropolitana a nivel de manzana. Disponible en: https:// www.inei.gob.pe/media/MenuRecursivo/publicaciones_digitales/Est/ Lib1403/index.html

8. Jara M. 2009. Bases para el establecimiento de un método de identificación de perros en la comuna de Maipú. Tesis de Médico Veterinario. Santiago de Chile: Univ. de Chile. 85 p.

9. Leguía G. 2002. Enfermedades parasitarias de perros y gatos. $2^{\circ} \mathrm{Ed}$. Lima, Perú: Ed De Mar. 155 p.

10. León D, Panta S, Yarlequé C, Falcón N. 2013. La convivencia con mascotas en zonas periurbanas: Experiencia en Lima - Perú. MV Rev Cien Vet 29: 21-25.

11. León D, Soriano JF, Arauco D, Falcón N. 2014. Estimación de la población de perros con dueño y perros vagabundos: importancia para la salud pública. MV Rev Cien Vet 30: 5-10.

12. Ley No. 27596 Régimen jurídico de canes. Diario El Peruano. 2002. p 225240-225244.

13. Llalla H. 2012. Caracterización de la tenencia de mascotas y evaluación de conocimientos, actitudes y prácticas asociadas a riesgos de zoonosis y accidentes entre escolares de educación secundaria en tres distritos de Lima Metropolitana. Tesis de Maestría. Lima, Perú: Univ. Peruana Cayetano Heredia. 75 p.

14. [MINSA] Ministerio de Salud. 2017. Norma técnica de salud para la prevención y control de la rabia humana en el Perúi. Lima: MINSA. Internet]. Disponible en: http://bvs.minsa.gob.pe/local/ DGSP/770_DGSP242.pdf

15. Recuenco S. 2019. Persistencia de la reemergencia de la rabia canina en el sur del Perú. Anales Fac Med 80: 379-382. 
16. Resolución Ministerial $N^{o}$ 1776-2002SA/DM. 2002. Consideran en la relación de razas de canes potencialmente peligrosos al Pit Bull Terrier, Dogo Argentino, Fila Brasilero,Tosa Japonesa, Bul Mastiff, Doberman y Rotweller. Internet]. Disponible en: https:// www.gob.pe/institucion/minsa/normaslegales/254511-1776-2002-sa-dm

17. Robertson I, Irwin P, Lymbery A, Thompson R. 2000. The role of companion animals in the emergence of parasitic zoonoses. Int J Parasitology 30: 1369-1377. doi: 10.1016/s0020-7519(00)-00134-x

18. Robles R, León D, Falcón N. 2017. Evaluación del contenido y aplicabilidad de las ordenanzas Municipales emitidas dentro del marco de la ley que regula el régimen jurídico de canes (Ley $\mathrm{N}^{\circ}$ 27596) en los distritos de Lima- Metropolitana. MV Rev Cienc Vet 33: 5-12.

19. Rojas P, León D, Falcón N. 2019. Características de los perros y gatos bajo control reproductivo quirúrgico registra- dos en la Municipalidad de Los Olivos, Lima, Perú. Periodo 2015-2016. Rev Inv Vet Perú 30: 818-827. doi: 10.15381/ rivep.v30i2.16093

20. Romero C, Hernández H, Falcón N. 2014. Costos de hospitalización de los accidentes por mordedura de canes en un hospital de niños en Lima-Perú, periodo 2006-2010. Salud Tecnol Vet 1:2632. doi: 10.20453/stv.v1i2.109

21. Serafini CA, Rosa GA, Guimaraes AM, De Morais HA, Biondo AW. 2008. Survey of owned feline and canine populations in apartments from a neighbourhood in Curitiba, Brazil. Zoonoses Public Hlth 55: 402-405. doi: 10.1111/j.1863-2378.2008.01171.x

22. Talavera M, Gamboa B, Gonzáles $J$, Huanambal C, León D, Falcón N. 2018. Accidentes por mordedura de canes y conocimiento de rabia urbana en pobladores de Madre de Dios y Puno, Perú, 2014. Rev Inv Vet Perú 29: 10251035. doi: 10.15381/rivep.v29i3.14837 